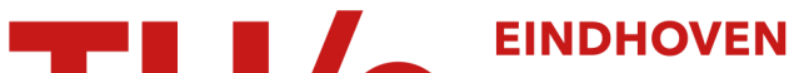 UNIVERSITY OF TECHNOLOGY
}

\section{Analytical models for the wake-up receiver power budget for wireless sensor networks}

\section{Citation for published version (APA):}

Lont, M., Milosevic, D., Dolmans, G., Baltus, P. G. M., \& Roermund, van, A. H. M. (2009). Analytical models for the wake-up receiver power budget for wireless sensor networks. In Proceeding of the IEEE Global

Telecommunications Conference, GLOBECOM 2009, November 30-December 4 2009, Honolulu, Hawai, USA, (pp. 1-6). Institute of Electrical and Electronics Engineers. https://doi.org/10.1109/GLOCOM.2009.5425470

DOI:

10.1109/GLOCOM.2009.5425470

Document status and date:

Published: 01/01/2009

\section{Document Version:}

Publisher's PDF, also known as Version of Record (includes final page, issue and volume numbers)

\section{Please check the document version of this publication:}

- A submitted manuscript is the version of the article upon submission and before peer-review. There can be important differences between the submitted version and the official published version of record. People interested in the research are advised to contact the author for the final version of the publication, or visit the DOI to the publisher's website.

- The final author version and the galley proof are versions of the publication after peer review.

- The final published version features the final layout of the paper including the volume, issue and page numbers.

Link to publication

\section{General rights}

Copyright and moral rights for the publications made accessible in the public portal are retained by the authors and/or other copyright owners and it is a condition of accessing publications that users recognise and abide by the legal requirements associated with these rights.

- Users may download and print one copy of any publication from the public portal for the purpose of private study or research.

- You may not further distribute the material or use it for any profit-making activity or commercial gain

- You may freely distribute the URL identifying the publication in the public portal.

If the publication is distributed under the terms of Article 25fa of the Dutch Copyright Act, indicated by the "Taverne" license above, please follow below link for the End User Agreement:

www.tue.nl/taverne

Take down policy

If you believe that this document breaches copyright please contact us at:

openaccess@tue.nl

providing details and we will investigate your claim. 


\section{Analytical Models for the Wake-up Receiver Power Budget for Wireless Sensor Networks}

\author{
Maarten Lont, Dusan Milosevic \\ Peter G.M. Baltus and Arthur H.M. van Roermund \\ Mixed-signal Microelectronics \\ Eindhoven University of Technology \\ Eindhoven, The Netherlands
}

\author{
Guido Dolmans \\ Holst Centre \\ Stichting IMEC-NL \\ Eindhoven, The Netherlands
}

\begin{abstract}
In this paper analytical models of the energy consumption are presented which uses a real world radio model with two different low power modes. This model is used to compare energy consumption of different MAC protocols. The MAC protocols used for the comparison are chosen with sensor networks is mind. The energy consumption of the nodes in a sensor network needs to be minimized to maximize the lifetime of the network. Emphasis is placed on MAC protocols, since they have a big influence on the energy consumption. One of the MAC protocols uses a low power Wake Up Receiver (WURx) which is used to decrease the total energy dissipation. The WURx MAC protocol is compared with two other low power MAC protocols, namely the asynchronous X-MAC and synchronous TDMA protocol. The obtained model is used to derive the WURx power budget. The response time of the nodes is used as the main design requirement and the important application parameters are given that determine the WURx power budget.
\end{abstract}

\section{INTRODUCTION}

Wireless sensor networks have many potential applications, for example medical body area networks (BAN). The vital signs of a patient can be monitored over a long time without the need for the patient to be physically in a hospital. Such a BAN network consists of a limited number of nodes and one of the main design goals is a low power consumption, since the nodes are battery powered making it inconvenient or even impossible to replace the batteries on a regular basis. Decreasing the radio energy consumption has a big impact on the total node energy consumption, since a large part of the energy is consumed by the radio [1].

One of the most important design constraint is the response time of a sensor node. In some applications, for example medical applications, data may have time limited relevance and has to be processed quickly, for example for pacemakers, and in control systems long latencies can lead to system instability [2]. In this paper MAC parameters are chosen in a way to satisfy a given response constraint.

A low power Wake Up Receiver (WURx) is added to a node, and it listens for radio transmissions while the main radio sleeps. This decreases the idle listening and overhearing power consumption, because the WURx has a significant smaller power consumption than the main radio. Overhearing means that a node receives packets that are destined to other nodes, see [3]. Furthermore the WURx MAC protocol is asynchronous and therefore has no synchronization overhead.

This paper presents an analytical model for the WURx power budget taking the application parameters into account and using a real world radio model. The conditions on the application parameters are analyzed that lead to a lower power consumption of a WURx system. The WURx power budget is obtained by comparing a MAC protocol with WURx to other MAC protocols.

MAC protocols can be divided in two groups, the asynchronous and synchronous schemes. B-MAC [4] and $X-M A C$ [5] are two examples of asynchronous lowpower, sensor-network, MAC protocols. The X-MAC scheme is shown to be more energy efficient because the transmitter does not have to send a long preamble, but several short preambles. This decreases the energy consumption caused by overhearing, see [5]. The X-MAC protocol can be used with the widely used packet radios in contrast to the B-MAC protocol.

Synchronous MAC protocols, like S-MAC [3], reduce the overhearing and idle listening power consumption by synchronizing the wake up periods. Nodes know when their neighbors are awake and only start the communication during this awake period. Furthermore the collisions can be reduced by appointing transmission slots, like in TDMA. The cost for this is the synchronization overhead and the resynchronization penalty. TDMA is a centrally coordinated synchronization scheme, unlike S-MAC. Static TDMA is beneficial for small networks since it moves the intelligence from the nodes to a central master node, which increases the node lifetime.

In section II a new and improved model is introduced, which is used to derive detailed new models for the energy consumption. These models are applied to various MAC protocols in section III. The application dependent energy consumption benefit of the WURx is analyzed in section $\mathrm{V}$ using the proposed models. The packet statistics are given in the appendix. 
TABLE I

RADIO MODEL PARAMETERS

\begin{tabular}{|l|l|}
\hline Parameter & Explanation \\
\hline \hline$\mu_{W U C}$ & WUC \\
$\mu_{A C K 1}$ & Initial ACKs \\
$\mu_{A C K x}$ & ACK retransmissions \\
$\mu_{\text {slot }}$ & TDMA slots needed per wake up attempt \\
\hline \hline$N^{+}$ & Maximal number of retransmission attempts \\
$k$ & ACK and WUC packet length \\
\hline
\end{tabular}

\section{Radio and Traffic Models}

Both a symmetric and asymmetric system are analyzed. In a symmetric system all the nodes in the network are equal and can both initiate and receive a data link setup. In an asymmetric system there is one master node which can initiate a data link setup, all the nodes are slaves. In this case the energy consumption of the master is less important than the energy consumption of a node since a master node can have a larger battery or can be connected to mains supply.

It is assumed that nodes can receive and transmit data to each other. The node which initiates a communication link is called here a transmitter and a node which receives a communication request is called a receiver. The radio parameters are summarized in table I.

Only the energy needed to set up a data link is taken into account since it is assumed that the data transfer itself is the same for the three MAC schemes used for the comparison: WURx, X-MAC and static TDMA. Furthermore, the application requires that the data link be set up within a given response time $T_{\text {response }}$.

A data link is set up in two steps, at the first step the transmitter transmits a Wake Up Call (WUC). When the WUC is received by the receiver it transmits an acknowledgment (ACK). Both the WUC and ACK are of minimal length $k$ and contain a destination address to decrease the overhearing problem. When the ACK is received by the transmitter the data link is set up and the data can be transferred.

There exist two types of errors, a packet can either be missed or falsely decoded. The packet miss probability is specified by $p_{\text {miss }}$ and a false wake up probability by $p_{\text {false }}$.

When the transmitter does not receive the ACK it retransmits the WUC maximally $N^{+}$times. A WUC consists of a preamble, a destination address and a counter which counts the number of setup attempts. The counter is used by the receiver to know how many attempts it has left to transmit the ACK. A receiver starts transmitting acknowledgments when a WUC is received and stops when either the maximal number of attempts is reached or the ACK is received by the transmitter. The packet statistics are analyzed in greater depth in the appendix. In table I the used average values are listed.

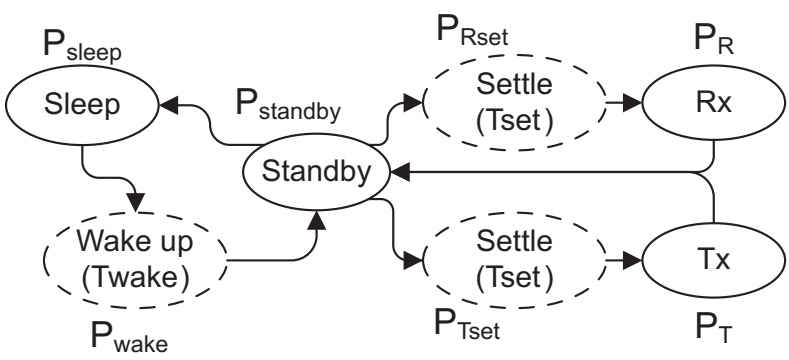

Fig. 1. Radio state diagram

\section{A. Radio States}

The radio state diagram is shown in figure 1 . The radio power consumption in each state is given by the parameters outside the state symbols. The dotted states are transition states, the time period a node stays in these states is given in parenthesis. The sleep mode is the lowest power mode, therefore $P_{\text {sleep }}<P_{\text {standby, }}$ and the energy consumed in each state is $E_{x}=T_{x} P_{x}$.

In the equations the difference in power and energy consumption of an active mode and the sleep mode are denoted by adding a leading $\Delta$.

\section{B. Traffic Scenario}

A network consist of $N_{\text {nodes }}$ nodes. It is assumed that each node receives the same number of packets with packet rate $\lambda$. The node energy consumption is normalized to the packet interval $\frac{1}{\lambda}$.

\section{MAC Energy Consumption Models}

The analytical models of the energy consumptions of three different MAC protocols are presented in the following sections. These models are used to obtain an analytical WURx power budget model.

\section{A. WURx MAC}

The WURx MAC scheme assumes an extra low power Wake Up Receiver added to the node. The bit rate $R_{b w}$ of the WURx can be different from the bit rate of the main radio, to decrease its power consumption $P_{\text {WURx }}$. The wake up cycle is shown in figure 2 (WURx cycle is not shown). To fulfill the response requirement the transmitter has to be able to transmit $N^{+}$WUCs within $T_{\text {response, }}$ this gives a WUC cycle period of $T_{\text {cycle }}=\frac{T_{\text {response }} T_{\text {wake }}}{N^{+}}$. In the shown example the first ACK is missed by the transmitter. From the figure a lower bound on $R_{b w}$ is obtained,

$$
R_{b w} \geq \frac{k}{T_{\text {WUC }}}=\frac{k}{T_{\text {cycle }}-2 T_{\text {set }}-T_{\text {wake }}-T_{A C K}}
$$

The maximum wake up duration of one packet is $T_{\text {response }}$ and it is assumed that each node receives the same number of packets. The receiver and transmitter average energy consumption per received packet are given by (2) and (3), respectively. The first term specifies 


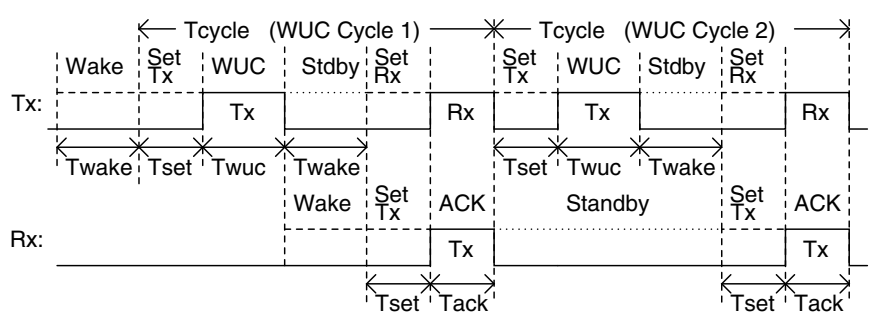

Fig. 2. WURx MAC layer

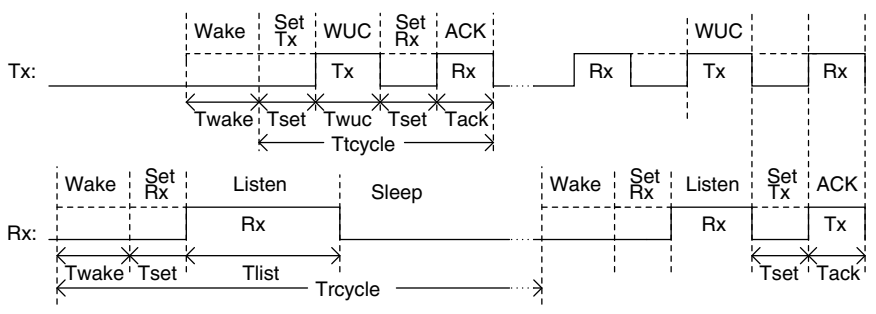

Fig. 3. X-MAC protocol

the energy consumption while sleeping, the second term is the energy spend on the initial ACK transmission and the third term gives the energy consumption for the retransmissions.

$$
\begin{aligned}
& E_{R x}=\frac{P_{W U R x}+P_{\text {sleep }}}{\lambda}+\mu_{A C K 1} E_{A C K 1}+\mu_{A C K x} E_{A C K x} \\
& E_{T x}=\mu_{\text {WUC }} E_{\text {WUC }}+\Delta E_{\text {wake }}
\end{aligned}
$$

where,

$$
\begin{aligned}
E_{\text {WUC }}= & \Delta E_{\text {Tset }}+T_{\text {WUC }} \Delta P_{T}+\Delta E_{\text {Rset }}+T_{A C K} \Delta P_{R}+ \\
& T_{\text {wake }} \Delta P_{\text {standby }} \\
E_{A C K 1}= & \Delta E_{\text {wake }}+\Delta E_{\text {Tset }}+T_{A C K} \Delta P_{T} \\
E_{A C K x}= & \left(T_{\text {wake }}+T_{\text {WUC }}+T_{\text {set }}\right) \Delta P_{\text {standby }}+\Delta E_{\text {Tset }}+T_{A C K} \Delta P_{T}
\end{aligned}
$$

\section{B. $X-M A C$}

The X-MAC [5] is the asynchronous MAC scheme used for comparison. The receiver wakes up periodically to listen for a WUC and the transmitter continuously transmits WUCs and listens for an ACK. The scheme is depicted in figure 3.

Since the receiver and transmitter are not synchronized the receiver has to listen long enough to receive two WUCs: it can happen that the receiver starts listening when the transmitter is already transmitting the wake up call. In that case the receiver will only receive the second one, using this the listening period $T_{\text {list }}$, see figure 3 , is given below.

$$
T_{\text {list }}=2 T_{\text {WUC }}+T_{A C K}+2 T_{\text {set }}
$$

The receiver has to be able to receive $N^{+}$WUCs within $T_{\text {response; }}$ t therefore, the receive cycle $T_{\text {rcycle }}$ is:

$$
T_{\text {rcycle }}=\frac{T_{\text {response }}}{N^{+}}
$$

The receiver listening duty cycle is given by $\eta$. A lower bound on $\frac{T_{\text {response }}}{N^{+}}$can be obtained using the fact that the duty cycle is maximally 1 .

$$
\eta=\frac{T_{\text {wake }}+T_{\text {set }}+T_{\text {list }}}{T_{\text {rcycle }}}
$$

The average number of WUC transmissions is given by $\mu_{\text {tcycle }}$ see (7). In this equation the first term gives the number of package needed to be send before the receiver receives a WUC, where the fact that the transmitter starts to transmit in the middle of the receiver cycle is. And the second term specifies the number of ACK that need to be retransmitted before the transmitter receives them.

$$
\mu_{\text {tcycle }}=\left(\mu_{\text {WUC }}-\frac{1}{2}\right) \frac{T_{\text {rcycle }}}{T_{\text {tcycle }}}+\mu_{A C K x}
$$

The receiver and transmitter energy consumptions are given by (8) and (9), respectively. The first two terms of the receiver specify the sleep mode and idle listening energy consumption, where $N_{\text {rcycle }}$ denotes the number of times per received packet the receiver listens. In the receiver equation the third and fourth term specify the energy consumption for transmitting the acknowledgments.

$$
\begin{aligned}
& E_{R x}=\frac{P_{\text {sleep }}}{\lambda}+N_{\text {rcycle }} E_{r c y c l e}+\mu_{A C K 1} E_{A C K 1}+\mu_{A C K x} E_{A C K x} \\
& E_{T x}=\mu_{\text {tcycle }} E_{\text {tcycle }}+\Delta E_{\text {wake }}
\end{aligned}
$$

where,

$$
\begin{aligned}
N_{\text {rcycle }} & =\frac{1}{\lambda T_{\text {rcycle }}} \\
E_{\text {rcycle }} & =\Delta E_{\text {wake }}+\Delta E_{\text {Rset }}+T_{\text {list }} \Delta P_{R} \\
E_{A C K 1} & =\Delta E_{\text {Tset }}+T_{A C K} \Delta P_{T} \\
E_{A C K x} & =\Delta E_{\text {Rset }}+T_{\text {WUC }} \Delta P_{R}+\Delta E_{\text {Tset }}+T_{A C K} \Delta P_{T} \\
E_{\text {tcycle }} & =\Delta E_{\text {Tset }}+T_{\text {WUC }} \Delta P_{T}+\Delta E_{\text {Rset }}+T_{A C K} \Delta P_{R}
\end{aligned}
$$

\section{Static TDMA}

With static TDMA the network consists of a Master node and one or more slave nodes; note that this is an asymmetric system. The master transmits a synchronization beacon every super frame. Within each super frame there can be a number of TDMA frames. Each TDMA frame has a slot for every node in the network; and the nodes can only communicate in their own slot. Figure 4 illustrates the MAC scheme where the sync period and one slot period are shown.

Each node has its own local clock with inaccuracy $\Theta[p p m]$ which leads to clock skew between two nodes. The maximally allowed clock skew is given by $T_{\text {skew }}$, which are depicted as gray areas in figure 4 . In this paper it is assumed that $T_{\text {beacon }} \gg \frac{T_{\text {response }}}{N^{+}}$, using this assumption the maximum time between two sync beacons $T_{\text {beacon }}$ to keep the whole network synchronized is given below.

$$
T_{\text {beacon }}(m s)=\frac{T_{\text {skerw }}(m s)}{\Theta(p p m)} 10^{6}
$$




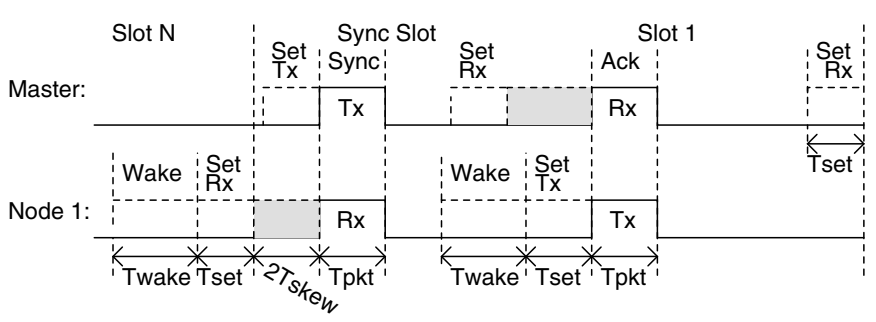

Fig. 4. Static TDMA MAC layer

The average number of beacons per received packet $\mu_{b c n / p k t}$ is given by (11).

$$
\mu_{b c n / p k t}=\frac{1}{\lambda T_{\text {beacon }}}
$$

When a node misses the synchronization beacon it stays in receive mode until it receives the next beacon in order to resynchronize. While resynchronizing all the packets are lost and the probability of this event is assumed to be equal to the packet miss probability. If $T_{\text {beacon }}$ is large the resynchronization penalty can be quite severe.

The node energy consumption is,

$$
E_{\text {Node }}=\frac{P_{\text {sleep }}}{\lambda}+\mu_{\text {bcn/pkt }} E_{\text {sync }}+p_{\text {miss }} \frac{\Delta P_{R}}{\lambda}+\mu_{\text {slot }} E_{\text {slot }}
$$

where,

$$
\begin{aligned}
E_{\text {sync }} & =\Delta E_{\text {wake }}+\Delta E_{\text {Rset }}+\left(2 T_{\text {skew }}+T_{\text {pkt }}\right) \Delta P_{R} \\
E_{\text {slot }} & =\Delta E_{\text {wake }}+\Delta E_{\text {Tset }}+T_{\text {pkt }} \Delta P_{T}
\end{aligned}
$$

\section{WURx Power Budget Model}

The WURx power budget is the difference in power consumption between the X-MAC or TDMA and the WURx MAC scheme. When the actual WURx power consumption is less than the budget the WURx scheme is more energy efficient. The approximated power budget is given below assuming $p_{\text {miss }}$ and $p_{\text {false }}$ are in the order of a few percent and can be neglected.

1) WURx vs X-MAC: The WURx power budgets are given by (13) and (14) for the asymmetric and symmetric systems, respectively. The power budget for the symmetric system consists of two terms, where the first term specifies the average power consumed by the receiver for the periodic listening and the second term gives the average power consumed for transmitting the WUCs by the transmitter.

$$
\begin{aligned}
P_{\text {WURx }, \text { asym }} & \lesssim \frac{N^{+}}{T_{\text {response }}} E_{\text {rcycle }}-\lambda \Delta E_{\text {wake }} \\
P_{\text {WUR } x \text {, sym }} & \lesssim P_{\text {WURx,asym }}+\lambda\left(\frac{T_{\text {response }}}{N^{+}} \frac{1}{2 T_{\text {tcycle }}}-1\right) E_{\text {tcycle }}
\end{aligned}
$$

2) WURx vs Static TDMA: Equation (15) gives the WURx power budget for an asymmetric system. It can be seen that the power budget is highly dependent on the resynchronization penalty, i.e. on $p_{\text {miss }}$. The WURx scheme can only be beneficial when the TDMA synchronization overhead is larger than the WURx overhead.

$$
P_{\text {WUR } x, \text { asym }} \lesssim \frac{E_{\text {sync }}}{T_{\text {beacon }}}+p_{\text {miss }} \Delta P_{R}
$$

3) $X-M A C$ vs Static TDMA: The TDMA scheme is more power efficient than the X-MAC scheme when $p_{\text {miss }}$ is lower than the bound given by (16).

$$
p_{\text {miss }} \leq \frac{\frac{N^{+}}{T_{\text {response }}} E_{\text {rcycle }}-\lambda \Delta E_{\text {wake }}-\frac{E_{\text {synce }}}{T_{\text {beacon }}}}{\Delta P_{R}}
$$

\section{Sleep Mode Boundary Conditions}

The sleep mode should be used when the penalty for waking up is lower than the energy consumption decrease. This can be written as,

$$
E_{\text {wake }} \leq E_{\text {standby }}-E_{\text {sleep }}
$$

The presented energy models assume that the sleep mode is used; when this is not the case then $T_{\text {wake }}=0$ and $E_{\text {sleep }}=E_{\text {standby }}$.

The boundary conditions for the sleep mode are derived for the different MAC protocols for asymmetric and symmetric systems. The assumption $\Delta P_{x} \approx P_{x}$ is used when deriving the boundary conditions.

\section{A. WURx}

The boundary condition on the packet rate is given below; when the inequality holds the sleep mode should be used.

$$
\begin{aligned}
\left.\lambda\right|_{\text {asymmetric }} & \lesssim \frac{P_{\text {standby }}-P_{\text {sleep }}}{E_{\text {wake }}} \\
\left.\lambda\right|_{\text {symmetric }} & \lesssim \frac{P_{\text {standby }}-P_{\text {sleep }}}{2 E_{\text {wake }}}
\end{aligned}
$$

\section{B. $X-M A C$}

For the asymmetric and symmetric X-MAC systems the boundary conditions for using sleep mode are:

$$
\begin{aligned}
\frac{N^{+}}{T_{\text {resp }}} & \leq \frac{P_{\text {standby }}-P_{\text {sleep }}}{E_{\text {wake }}} \\
\left.\lambda\right|_{\text {symmetric }} & \leq \frac{P_{\text {standby }}-P_{\text {sleep }}}{E_{\text {wake }}}-\frac{N^{+}}{T_{\text {resp }}}
\end{aligned}
$$

The boundary condition on the response requirement holds for both types of systems, whereas the bound on the packet rate exists only for the symmetric system.

\section{Static TDMA}

The static TDMA scheme can only be used in an asymmetric system. The sleep mode should be used when the inequality given below holds.

$$
\lambda \leq \frac{P_{\text {standby }}-P_{\text {sleep }}}{E_{\text {wake }}}-\frac{1}{T_{\text {beacon }}}
$$




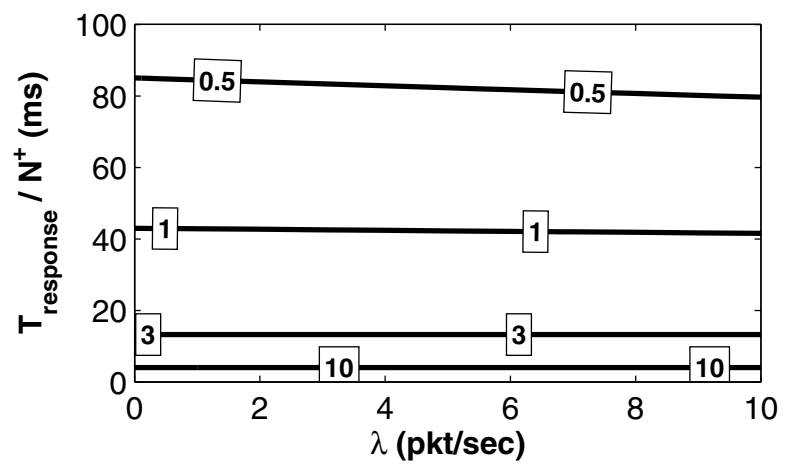

Fig. 5. Boundary condition on $p_{\text {miss }}(\%)$, given the packet rate $\lambda$ and response time $\frac{T_{\text {response }}}{N^{+}}$.

\section{Application Dependent Energy Consumption}

To obtain the numerical results in this section the Nordic nRF24L01 [6] radio is used. The radio parameters are given in table II(a) and the application parameters are given in table II(b). In this section the results are given as a function of the important application parameters: $\frac{T_{\text {response }}}{N^{+}}$and $\lambda$.

TABLE II

MODEL PARAMETERS

(a) nRF24L01

\begin{tabular}{|l|c|}
\hline Parameter & Value \\
\hline$T_{\text {wake }}$ & $1.5 \mathrm{~ms}$ \\
$T_{\text {set }}$ & $130 \mu \mathrm{s}$ \\
\hline$P_{\text {sleep }}$ & $2.7 \mu \mathrm{W}$ \\
$P_{\text {standby }}$ & $66 \mu \mathrm{W}$ \\
$P_{R}$ & $36.9 \mathrm{~mW}$ \\
$P_{T}$ & $33.9 \mathrm{~mW}$ \\
$P_{\text {Rset }}$ & $25.2 \mathrm{~mW}$ \\
$P_{\text {Tset }}$ & $24 \mathrm{~mW}$ \\
$P_{\text {wake }}$ & $855 \mu \mathrm{W}$ \\
\hline Packet size $k$ & $34 \mathrm{bits}$ \\
Bit rate $R_{b}$ & $2 \mathrm{Mbps}$ \\
$\Theta$ & $50 \mathrm{ppm}$ \\
\hline
\end{tabular}

(b) Application

\begin{tabular}{|l|c|}
\hline Parameter & Value \\
\hline$N_{\text {nodes }}$ & 12 \\
$N^{+}$ & 3 \\
\hline$p_{\text {miss }}$ & $1 \%$ \\
$p_{\text {false }}$ & $1 \%$ \\
\hline
\end{tabular}

If the packet miss probability is lower than the bound given by (16) and depicted in figure 5 then the TDMA protocol is more power efficient than the X-MAC protocol. The X-MAC system has to wake up less often when the required response time is higher. The TDMA protocol is more energy efficient for high response times when the TDMA resynchronization penalty is low. This can be seen in figure (5), for high response times the boundary condition on $p_{\text {miss }}$ is lower.

The WURx power budget for an asymmetric system as function of the packet rate $\lambda$ and $\frac{T_{\text {response }}}{N^{+}}$is shown in figure 6, where the dotted line shows the approximated and the solid line the actual power budget. The approximated power budgets were given by (13) and (15) for the X-MAC and TDMA protocols, respectively. It can be seen that the approximations are good, thus the $p_{\text {miss }}$ and $p_{\text {false }}$ can be neglected when they are smaller than a few

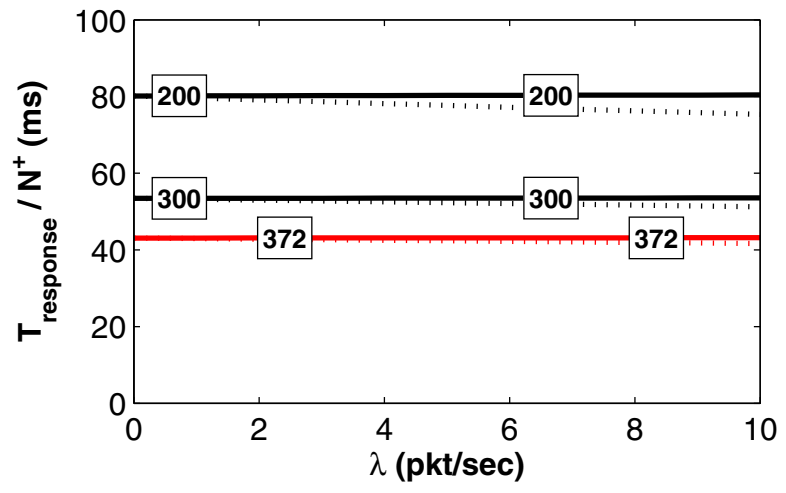

Fig. 6. WURx power budget $(\mu \mathrm{W})$ for an asymmetric system given the application parameters.

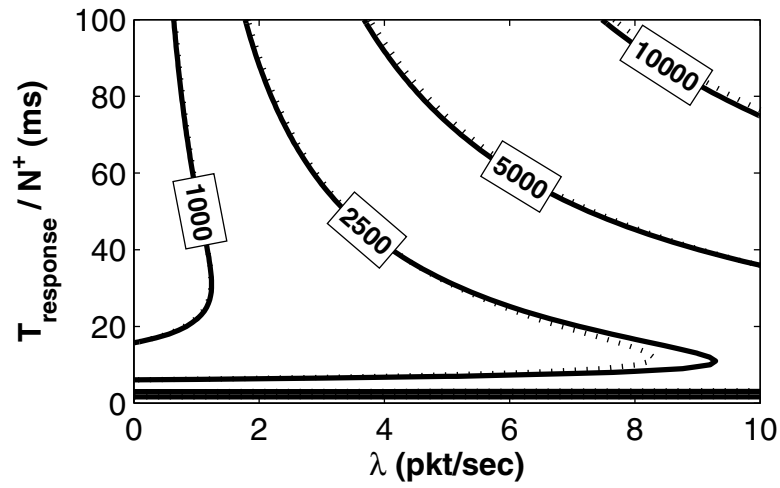

Fig. 7. WURx power budget $(\mu \mathrm{W})$ for a symmetric system as function of the application parameters $\lambda$ and $\frac{T_{\text {response }}}{N^{+}}$.

percent. For high response times the WURx protocol is compared to the X-MAC protocol since it is more power efficient than the TDMA protocol. Since the X-MAC node can sleep for longer periods for high response times the power budget is lower. The budget decreases for higher packet rate, because the WURx receiver needs to wake up from sleep mode once per packet reception. For $p_{\text {miss }}=1 \%$ the TDMA scheme is more power efficient when $\frac{T_{\text {response }}}{N^{+}} \lesssim 40 \mathrm{~ms}$, as can be seen in figure 5 . This boundary condition is shown by the line labeled with a power budget of $372 \mu \mathrm{W}$.

The WURx power budget of a symmetric system is shown in figure 7. Again, the approximation is shown by the dotted lines and the real power budget is shown by the solid lines. The TDMA system can not be used as a symmetric system, as there is always a master node, thus the WURx energy consumption can only be compared to the X-MAC protocol. Since the X-MAC transmitter needs to transmit many WUCs before the receiver wakes up the power budget for the symmetric system is much larger than for the asymmetric system. When the packet rate is fixed there are two possible response times for a given power budget. The power consumption is mainly determined by the transmitter 
for the large response time. For the smaller response time the periodic listening power consumption of the receiver determines the power budget. At the boundary between the two regions the transmitter and receiver contributions are equal.

For large $T_{\text {response }}$ the power budget increases for higher response times since the $\mathrm{X}$-MAC transmitter needs to transmit more WUCs which costs a lot of energy. For lower response times, where the idle listening power consumption is significant, a decrease in response time leads to an increase in power budget. The power budget increases with an increase of the packet rate, because the $\mathrm{X}-\mathrm{MAC}$ transmitter transmits more WUCs.

\section{Conclusions}

This paper presents analytical models for the energy consumption of three different MAC protocols as well as a WURx MAC protocol. Using the analytical models the application dependent maximum power budget for the WURx is obtained for asymmetric and symmetric systems. The used radio model has a deep sleep mode and a low power standby mode. Whether the deep sleep mode should be used can be decided when the given parameters of the actual radio are known.

The TDMA protocol is more power efficient than the $\mathrm{X}$-MAC protocol when the response requirement and the packet miss probability are low. For asymmetric systems the WURx MAC protocol is most efficient when the response time requirement is low. For the symmetric system the WURx MAC protocol is most efficient when the response time and the packet rate is high.

\section{ApPEndix \\ Packet statistics}

The expected number of WUC and ACK transmissions are calculated using (23). Where $p_{x, k}$ is the probability on $\mathrm{k}$ number of $\mathrm{x}$ transmission attempts.

$$
\mu_{x}=\sum_{k=1}^{N_{W U C}^{+}} k p_{x, k}
$$

\section{A. Wake up calls}

The probability of $\mathrm{n}$ WUC transmissions $p_{W U C, n}$ is,

$$
p_{W U C, n}=n\left(1-p_{\text {miss }}\right)^{2} p_{\text {miss }}^{n-1}
$$

The receiver starts transmitting ACK after it receives any of the $n$ WUC packets. The transmitter stops transmitting after it correctly receives an ACK. For the other $n-1$ wake up attempts the WUC or ACK packet is missed. An error is made for $p_{W U C, N_{W U C}^{+}}$, since the transmitter stops after $N_{W U C}^{+}$attempts regardless whether it was successful or not.

$$
p_{W U C, N_{\text {WUC }}^{+}}=1-\sum_{k=1}^{N_{\text {WUC }}^{+}-1} p_{W U C, k}
$$

\section{B. Acknowledgments}

The probability distribution of $n$ correct ACK transmissions $p_{A C K, C o r r e c t, n}$ is given below. The first term specifies the events where both the WUC and ACK are received correctly. The second term specifies the probability that all the acknowledgments are missed.

$$
p_{A C K, \text { correct }, n}=\left(1-p_{\text {miss }}\right)^{2} \sum_{k=n}^{N_{\text {WUC }}^{+}} p_{\text {miss }}^{k-1}+\left(1-p_{\text {miss }}\right) p_{\text {miss }}^{N_{\text {WuC }}^{+}}
$$

The false wake up probability per other node in the network is given by (27). The first $N_{W U C}^{+}-n$ WUC packets are received correctly. The summation gives the probability that more than $N_{W U C}^{+}-n$ WUCs are transmitted.

$$
p_{\text {ACK, false } n}=p_{\text {false }}\left(1-p_{\text {false }}\right)^{N_{W U C}^{+}-n} \sum_{k=N_{W U C}^{+}-n+1}^{N_{W U C}^{+}} p_{W U C, n}
$$

The ACK transmission probability distribution is given below. The receiver can falsely wake up when one of the other nodes transmits WUCs.

$$
p_{A C K, n}=p_{A C K, \text { correct }, n}+\left(N_{\text {nodes }}-1\right) p_{A C K, \text { false }, n}
$$

The expected number of initial ACK transmissions $\left(\mu_{A C K 1}\right)$ and retransmissions $\left(\mu_{A C K x}\right)$ are given by,

$$
\begin{aligned}
& \mu_{A C K 1}=p_{A C K, \text { correct }, 1}+\left(N_{\text {nodes }}-1\right) p_{A C K, \text { false }, 1} \\
& \mu_{A C K x}=\mu_{A C K}-\mu_{A C K 1}
\end{aligned}
$$

C. TDMA slots

The probability that a node needs $n$ TDMA slots to transmit an ACK $p_{\text {slot }, n}$ is,

$$
p_{\text {slot }, n}=\left(1-p_{\text {miss }}\right) p_{\text {miss }}^{n-1}
$$

The node stops transmitting after the master receives the ACK. For the other $n-1$ attempts the ACK packet is missed. An error is made for $p_{\text {slot }, N_{W u C}^{+}}$since the node stops after $N_{W U C}^{+}$attempts.

$$
p_{\text {slot }, N_{\text {WUC }}^{+}}=1-\sum_{k=1}^{N_{\text {WUC }}^{+}-1} p_{\text {slot }, k}
$$

\section{REFERENCES}

[1] W. Ye, F. Silva, and J. Heidermann, "Ultra-low Duty Cycle MAC with Scheduled Channel Polling," Proc. Sensys 2006, pp. 321-334, 2006.

[2] A. Belmiloudi, Stabilization, Optimal and Robust Control. Upper Saddle River, NJ 07458: Springer, 2008.

[3] W. Ye, J. Heidermann, and D. Estrin, "An Energy-Efficient MAC Protocol for Wireless Sensor Networks," INFOCOM 2002, pp. 15671576, June 2002

[4] J. Polastre, J. Hill, and D. Culler, "Versatile Low Power Media Access for Wireless Sensor Networks," The Second ACM Conference on Embedded Networked Sensor Systems, pp. 95-107, 2004.

[5] M. Buettner, G. Yee, E. Anderson, and R. Han, "X-MAC A Short Preamble MAC Protocol for Duty-Cycled Wireless Sensor Networks," ACM Conference on Embedded Sensor Systems, 2006.

[6] Single Chip 2.4GHz Transceiver nRF24L01, Nordic Semiconductor, http://www.nordicsemi.com/index.cfm?obj=product $\backslash \&$ act $=$ display $\backslash \&$ pro $=89,2008$. 